\title{
Dependência Espacial de Funções: uma Generalização
}

M.C. FENILLE ${ }^{1}$, Instituto de Ciências Matemáticas e de Computação, USP, 13560970 São Carlos, SP, Brasil.

Resumo Este trabalho consiste de uma generalização dos conceitos de dependência espacial trazidos do âmbito da Geoestatística para uma abordagem puramente matemática nos casos em que se tem definida uma função contínua a valores reais sobre um domínio consistindo de uma região do espaço Euclideano $m$-dimensional.

\section{Dependência $\mu$-espacial}

Seja $\mathbb{R}^{m}, m \geq 2$, o espaço Euclideano $m$-dimensional munido da métrica usual $d$ e da medida de Lebesgue $\mu$. Diremos que um subconjunto $\mathbb{D} \subset \mathbb{R}^{m}$ é uma região se $\mathbb{D}$ é um $m$-disco fechado localmente plano, ou seja, $\mathbb{D}$ é homeomorfo a um $m$-disco fechado e para todo ponto $x$ da fronteira $\partial \mathbb{D}$ de $\mathbb{D}$, existe uma $m$-bola aberta $B_{r}^{m}(x)$ tal que $\partial \mathbb{D} \cap B_{r}^{m}(x)$ é homeomorfo a uma $(m-1)$-bola aberta.

Seja $E$ um subconjunto $k$-dimensional do $\mathbb{R}^{m}$ com $0 \leq k \leq m$. Vamos escrever $v_{k}(E)$ para denotar a medida de Lebesgue (ou volume) $k$-dimensional de $E$. No caso particular $k=0$, escrevemos $v_{0}(E)=\# E$ para indicar a cardinalidade de $E$.

Em todo o texto, $f: \mathbb{D} \rightarrow \mathbb{R}$ será uma função contínua a valores reais arbitrariamente definida numa região $\mathbb{D} \subset \mathbb{R}^{m}$. Por compacidade, $f$ é limitada.

Escrevemos $\delta(\mathbb{D})$ para denotar o diâmetro da região $\mathbb{D}$, e $\mathcal{H}=[0, \delta(\mathbb{D})]$. Para cada $h \in \mathcal{H} \backslash\{0\}$ e cada $x \in \mathbb{D}$, denotaremos $\mathbb{D}_{h}(x)=\bar{B}_{h}(x) \cap \mathbb{D}$, onde $\bar{B}_{h}(x)$ é a $m$-bola fechada de centro em $x$ e raio $h$. Convencionalmente $\mathbb{D}_{0}(x)=\{x\}$. Denotamos, ainda,

$$
\mathbb{S}_{h}(x)=\partial B_{h}(x) \cap \mathbb{D} .
$$

Observe que $\mathbb{S}_{h}(x)$ consiste de um subconjunto da $(m-1)$-esfera de centro em $x$ e raio $h$ contida no espaço $\mathbb{R}^{m}$, exceto o caso particular convencionado $\mathbb{S}_{0}(x)=\{x\}$. Agora, note que o conjunto $\mathbb{S}_{h}(x)$ pode não ser conexo. No entanto, pela definição de região, $\mathbb{S}_{h}(x)$ possuirá somente um número finito de componentes conexas.

Sobre o conjunto $\mathcal{H} \times \mathbb{D}$ consideramos a estrutura de topologia produto onde $\mathcal{H}$ e $\mathbb{D}$ têm as topologias induzidas das topologias usuais de $\mathbb{R}$ e $\mathbb{R}^{m}$, respectivamente.

Para cada par $(h, x) \in \mathcal{H} \times \mathbb{D}$ e cada número real $\varepsilon>0$, o conjunto $V_{\varepsilon}(h, x)=$ $\left\{(r, y) \in \mathcal{H} \times \mathbb{D}: r \in(h-\varepsilon, h+\varepsilon), y \in B_{\varepsilon}(x)\right\}$ constitue uma vizinhança aberta de $(h, x)$ em $\mathcal{H} \times \mathbb{D}$, a qual chamar-se-á a $\varepsilon$-vizinhança de $(h, x)$ em $\mathcal{H} \times \mathbb{D}$.

\footnotetext{
${ }^{1}$ fenille@icmc.usp.br
} 
Seja $\mathcal{P}_{\mathbb{S}}(\mathbb{D})$ o subconjunto das partes de $\mathbb{D}$ constituído exclusivamente pelos subconjuntos $\mathbb{S}_{h}(x)$, com $h \in \mathcal{H}$ e $x \in \mathbb{D}$. Munimos este conjunto de uma estrutura topológica como se segue: definimos a $\varepsilon$-vizinhança de $\mathbb{S}_{h}(x)$ em $\mathcal{P}_{\mathbb{S}}(\mathbb{D})$ como sendo o conjunto $V_{\varepsilon}\left(\mathbb{S}_{h}(x)\right)=\left\{\mathbb{S}_{r}(y):(r, y) \in V_{\varepsilon}(h, x)\right\}$. Quando $\varepsilon$ percorre os números reias positivos, os conjuntos $V_{\varepsilon}\left(\mathbb{S}_{h}(x)\right)$ descrevem uma base fundamental de vizinhanças para $\mathbb{S}_{h}(x)$ em $\mathcal{P}_{\mathbb{S}}(\mathbb{D})$. A topologia gerada por esta base denominar-se-á a $\mathcal{P}_{\mathbb{S}}$-topologia induzida de $\mathcal{H} \times \mathbb{D}$ em $\mathcal{P}_{\mathbb{S}}(\mathbb{D})$.

Todas estas estruturas e terminologias não serão requeridas ou novamente mencionadas explicitamente no restante do texto, mas sua introdução aqui vem à garantir a fundamentação matemática de argumentos muito importantes sobre os quais se baseia o restante do trabalho. Falamos, na verdade, do primeiro lema, abaixo enunciado, o qual tem sua demonstração inteiramente garantida pelo simples fato de termos, nas notações do lema, a igualdade de conjuntos $\mathbb{S}^{-1}\left(V_{\varepsilon}\left(\mathbb{S}_{h}(x)\right)\right)=V_{\varepsilon}(h, x)$.

Lema 1.1. A função $\mathbb{S}: \mathcal{H} \times \mathbb{D} \rightarrow \mathcal{P}_{\mathbb{S}}(\mathbb{D})$ definida por $\mathbb{S}(h, x)=\mathbb{S}_{h}(x)$ é contínua.

Chamamos volume circular local a função

$$
\vartheta: \mathcal{H} \times \mathbb{D} \rightarrow \mathbb{R} \text { dada por } \vartheta(h, x)=v_{m-1}\left(\mathbb{S}_{h}(x)\right) .
$$

De posse desta, denominamos volume circular a função global em $\mathbb{D}$

$$
\mathcal{N}: \mathcal{H} \rightarrow \mathbb{R} \text { dada por } \mathcal{N}(h)=\frac{1}{2} \int_{\mathbb{D}} \vartheta(h, x) d \mu(x)
$$

Uma vez considerada uma função contínua $f: \mathbb{D} \rightarrow \mathbb{R}$, definimos a função denominada $\mu$-variância circular local

$$
\mathcal{M}_{f}: \mathcal{H} \times \mathbb{D} \rightarrow \mathbb{R} \text { por } \mathcal{M}_{f}(h, x)=\int_{\mathbb{S}_{h}(x)}|f(x)-f(y)|^{2} d \mu(y)
$$

Observe que o valor $\mathcal{M}_{f}(h, x)$ fica bem definido ainda que $\mathbb{S}_{h}(x)$ não seja conexo. De fato, se $\mathbb{S}_{h}(x)$ possui $k$ componentes conexas, digamos $\mathbb{S}_{h}^{1}(x), \cdots, \mathbb{S}_{h}^{k}(x)$, então

$$
\mathcal{M}_{f}(h, x)=\sum_{i=1}^{k} \int_{\mathbb{S}_{h}^{i}(x)}|f(x)-f(y)|^{2} d \mu(y) .
$$

Com base no lema anterior e no fato de serem $\mathcal{H}$ e $\mathbb{D}$ espaços compactos, torna-se trivial a demonstração do seguinte.

Lema 1.2. As funções $\vartheta, \mathcal{N}$ e $\mathcal{M}_{f}$ são todas contínuas, limitadas e não-negativas.

Este resultado será muito importante daqui em diante, uma vez que definiremos uma função que envolverá um quociente com denominador $2 \mathcal{N}$ e numerador envolvendo $\mathcal{M}_{f}$. Mas para tanto, precisamos que $\mathcal{N}$ não possua zeros em $\mathcal{H}$. Mas isto não ocorre deveras. Não obstante, temos o seguinte resultado:

Proposição 1.1. $\mathcal{N}(h)=0$ se, e somente se, $h=0$ ou $h=\delta(\mathbb{D})$. 
Demonstração. Para todo ponto $x \in \mathbb{D}$ tem-se $\vartheta(0, x)=0$, já que $\mathbb{S}_{0}(x)=\{x\}$. Logo, $\mathcal{N}(0)=0$. Agora, seja $A=\{x \in \mathbb{D}: \exists y \in \mathbb{D} \operatorname{com} d(x, y)=\delta(\mathbb{D})\} \subset \mathbb{D}$. Neste caso, é claro que $A \subset \partial \mathbb{D}$ e, portanto, $v_{m}(A)=0$. Sendo assim, tem-se

$$
\mathcal{N}(\delta(\mathbb{D}))=\frac{1}{2} \int_{\mathbb{D}} \vartheta(\delta(\mathbb{D}), x) d \mu(x)=\frac{1}{2} \int_{A} \vartheta(\delta(\mathbb{D}), x) d \mu(x)=0 .
$$

Por outro lado, considere $h \in(0, \delta(\mathbb{D}))$ arbitrariamente fixado. Ainda no conjunto $A$ acima definido, fixe um elemento $a$. Seja $\varepsilon>0$ um número real, com $\varepsilon<\delta(\mathbb{D})-h$. É fácil ver que $v_{m}\left(\mathbb{D}_{\varepsilon}(a)\right)>0$ e, além disso, para todo $x \in \mathbb{D}_{\varepsilon}(a)$, o conjunto $\mathbb{S}_{h}(x)$ é uma reunião de arcos, sendo ao menos um deles não-degenerado. Logo, $\vartheta(h, x)>0$ para todo $x \in \mathbb{D}_{\varepsilon}(a)$ e, sendo $v_{m}\left(\mathbb{D}_{\varepsilon}(a)\right)>0$, segue-se que

$$
\mathcal{N}(h)=\frac{1}{2} \int_{\mathbb{D}} \vartheta(\delta(\mathbb{D}), x) d \mu(x) \geq \frac{1}{2} \int_{\mathbb{D}_{\varepsilon}(a)} \vartheta(\delta(\mathbb{D}), x) d \mu(x)>0 .
$$

Passamos agora à definição e estudo da seguinte função:

$$
\gamma_{f}^{\diamond}: \mathcal{H} \backslash\{0, \delta(\mathbb{D})\} \rightarrow \mathbb{R} \text { dada por } \gamma_{f}^{\diamond}(h)=\frac{1}{2 \mathcal{N}(h)} \int_{\mathbb{D}} \mathcal{M}_{f}(h, x) d \mu(x)
$$

Proposição 1.2. A função $\gamma_{f}^{\diamond}$ é contínua.

Demonstração. Pelo Lema 1.2 as funções $\mathcal{M}_{f}$ e $\mathcal{N}$ são ambas contínuas e limitadas. Além disso, pela Proposição $1.1, \mathcal{N}(h) \neq 0$ para todo $h \in \mathcal{H} \backslash\{0, \delta(\mathbb{D})\}$. Logo, como $\mathbb{D}$ é compacto, para qualquer $h_{0} \in \mathcal{H} \backslash\{0, \delta(\mathbb{D})\}$ tem-se

$$
\lim _{h \rightarrow h_{0}} \gamma_{f}^{\diamond}=\lim _{h \rightarrow h_{0}} \frac{1}{2 \mathcal{N}(h)} \int_{\mathbb{D}} \lim _{h \rightarrow h_{0}} \mathcal{M}_{f}(h, x) d x=\frac{1}{2 \mathcal{N}\left(h_{0}\right)} \int_{\mathbb{D}} \mathcal{M}_{f}\left(h_{0}, x\right) d x=\gamma_{f}^{\diamond}\left(h_{0}\right) .
$$

Gostaríamos de estender continuamente $\gamma_{f}^{\diamond}$ a uma função $\gamma_{f}$ definida também na origem. A realização de tal desejo é garantida através do seguinte resultado.

Proposição 1.3. Existe e é real o limite $\lim _{h \rightarrow 0} \gamma_{f}^{\diamond}$.

Demonstração. Note inicialmente que se $f: \mathbb{D} \rightarrow \mathbb{R}$ é uma função constante em quase toda parte, então, por continuidade, $f$ é constante. Logo $\mathcal{M}_{f}$ é a função nula e, neste caso, $\lim _{h \rightarrow 0} \gamma_{f}^{\diamond}=0$.

Assumamos, então, que $f: \mathbb{D} \rightarrow \mathbb{R}$ não seja constante em quase toda parte. Fixe $h \in \mathcal{H} \backslash\{0\}$ arbitrariamente pequeno. Como $f$ é contínua, existem $x$ no interior de $\mathbb{D}$ e um aberto $V$ de $\mathbb{D}$, tais que $V \cap \mathbb{S}_{h}(x)$ contém um arco não-degenerado e $|f(x)-f(y)|^{2}>\varepsilon$ para todo $y$ em um subconjunto $U \subset V \cap \mathbb{S}_{h}(x), \operatorname{com} v_{m-1}(U)>0$, onde $\varepsilon>0$ é um número real. Como, por outro lado, $f$ é limitada, existe um real $k>0$ tal que $|f(y)-f(x)|^{2} \leq k$, para todo $y \in \mathbb{D}$. Segue-se, então, as desigualdades

$$
\varepsilon v_{m-1}(U) \leq \mathcal{M}_{f}(h, x) \leq k v_{m-1}\left(\partial B_{h}(0)\right) .
$$

Prosseguindo com $h \in \mathcal{H} \backslash\{0\}$ inicial e arbitrariamente fixado, considere o conjunto $E_{h}=\{x \in \mathbb{D}: d(x, \partial \mathbb{D}) \leq h\}$. Se $h$ é suficientemente pequeno, então 
$v_{m}\left(E_{h}\right)>0$. Além disso, para todo $x \in E_{h}$, tem-se $\mathbb{S}_{h}(x)=\partial B_{h}(x)$. Se, por outro lado, $x \notin E_{h}$, então $\mathbb{S}_{h}(x) \varsubsetneqq \partial \bar{B}_{h}(x)$. Segue-se as desigualdades

$$
\frac{1}{2} v_{m}\left(E_{h}\right) v_{m-1}\left(\partial B_{h}(0)\right) \leq \mathcal{N}(h) \leq \frac{1}{2} v_{m}(\mathbb{D}) v_{m-1}\left(\partial B_{h}(0)\right),
$$

Agora, como $v_{m}\left(E_{h}\right) \rightarrow v_{m}(\mathbb{D})$ quando $h \rightarrow 0$, segue-se das inequações 1.1 e 1.2 que o limite $\lim _{h \rightarrow 0} \gamma_{f}^{\diamond}(h)$ existe e é um número real.

Doravante denotaremos $\mathcal{H}_{\mathbb{D}}=\mathcal{H} \backslash\{\delta(\mathbb{D})\}$. Pela proposição acima, podemos estender continuamente a função $\gamma_{f}^{\diamond}(h)$ ao domínio $\mathcal{H}_{\mathbb{D}}$ como se segue.

Definição 1.1. Chamamos $\mu$-semivariograma induzido por $f$ a função

$$
\gamma_{f}: \mathcal{H}_{\mathbb{D}} \rightarrow \mathbb{R} \text { dada por } \gamma_{f}(h)=\left\{\begin{array}{ccc}
\lim _{h \rightarrow 0} \gamma_{f}^{\diamond} & \text { se } & h=0 \\
\gamma_{f}^{\diamond} & \text { se } & h \neq 0
\end{array} .\right.
$$

Teorema 1.1. A função $\gamma_{f}$ é contínua e não-negativa.

Demonstração. Segue diretamente da Proposição 1.3 e da Definição 1.1.

Definição 1.2. Diremos que uma função contínua $f: \mathbb{D} \rightarrow \mathbb{R}$ é $\mu$-espacialmente dependente se existe um número real $a$, com $0<a \leq \delta(\mathbb{D})$, tal que $\gamma_{f}$ é estritamente crescente em $[0, a)$. O supremo dos números reais com esta propriedade será denominado alcance da dependência $\mu$-espacial de $f$ e denotado por $\mathfrak{a}$.

Exemplo 1.1. Sejam $\mathbb{D} \subset \mathbb{R}^{m}$ uma região e $f: \mathbb{D} \rightarrow \mathbb{R}$ uma função contínua constante em quase toda parte. Então f é constante e não é $\mu$-espacialmente dependente.

Apesar da consistência da teoria até aqui desenvolvida, é bastante difícil se determinar, segundo a definição acima, se uma dada função $f: \mathbb{D} \rightarrow \mathbb{R}$ é ou não é $\mu$-espacialmente dependente, a menos, é claro, que ela seja uma função constante em quase toda parte. E a dificuldade não reside apenas na complexidade que possa existir na lei que define a função $f$, mas sobretudo na complexidade da região $\mathbb{D}$.

Na seção seguinte estudaremos um caso especial da generalização a que se propõe este trabalho, e na seção 3 estudaremos maneiras para se tornar esta teoria de prática mais viável.

\section{O Caso Especial 1-dimensional}

Se considerarmos uma função contínua $f: \mathbb{D} \rightarrow \mathbb{R}$ definida em uma região $\mathbb{D}$ do espaço real 1-dimensional, veremos que alguns resultados já apresentamos neste texto passam a não ser verdadeiros em geral. É o caso, por exemplo, da função $\vartheta$ que jamais será contínua, neste contexto. Não obstante, podemos realizar o estudo da dependência $\mu$-espacial de $f$ de um modo bastante geral, como se segue.

Seja $\mathbb{D} \subset \mathbb{R}$ uma região. Então, $\mathbb{D}$ não pode ser outra coisa senão um intervalo fechado, digamos $\mathbb{D}=[a, b]$, com $a<b$. Uma esfera de centro em $x$ e raio $r>0$ em 
$\mathbb{R}$ é um conjunto constituído por somente dois pontos. Neste caso, para $h \in \mathcal{H}=$ $[0, b-a], h \neq 0$, e $x \in \mathbb{D}$, podemos ter $\vartheta(h, x)=0,1$ ou 2. Mais especificamente,

$$
\vartheta(h, x)=\left\{\begin{array}{lll}
0 & \text { se } & h>\max \{x-a, b-x\} \\
1 & \text { se } & \min \{x-a, b-x\}<h \leq \max \{x-a, b-x\} \\
2 & \text { se } & h \leq \min \{x-a, b-x\}
\end{array}\right.
$$

Se mantemos a convenção $\mathbb{S}_{0}(x)=\{x\}$, vemos que $\vartheta(0, x)=1$ para todo $x \in \mathbb{D}$, o que deve gerar oposição, neste caso específico, ao resultado da Proposição 1.1. De fato, considerado isto, tem-se $\mathcal{N}(0)=b-a$. Apesar disso manteremos a convenção e veremos que tais oposições aos resultados da seção 1 não serão pertinentes ao caso 1-dimensional agora tratado.

Segue da expressão encontrada para $\vartheta(h, x)$, através de um cálculo simples, que $\mathcal{N}: \mathcal{H} \rightarrow \mathbb{R}$ fica determinada pela lei $\mathcal{N}(h)=b-a-h$. Obtemos assim uma forma geral para a função $\mathcal{N}$, que é sempre contínua, apesar de $\vartheta$ não sê-lo.

Seja $f: \mathbb{D} \rightarrow \mathbb{R}$ uma função contínua. Tem-se, então, $\mathcal{M}_{f}(h, x): \mathcal{H} \times \mathbb{D} \rightarrow \mathbb{R}$ definida como $\mathcal{M}_{f}(h, x)=\sum_{y \in \mathbb{S}_{h}(x)}|f(x)-f(y)|^{2}$, onde o conjunto $\mathbb{S}_{h}(x)$ ou é vazio ou consiste de um ponto $(y=x+h$ ou $y=x-h)$ ou de dois pontos $(y=x+h \mathrm{e}$ $y=x-h)$. Logo, a determinação de $\mathcal{M}_{f}(h, x)$ é bastante simples. Além disso $\mathcal{M}_{f}$ apresentará no máximo dois pontos de descontinuidade.

Por fim, a função $\gamma_{f}: \mathcal{H} \rightarrow \mathbb{R}$ fica bem definida como $\gamma_{f}(h)=\int_{a}^{b} \mathcal{M}_{f}(h, x) d(x)$, havendo sequer a necessidade de defini-la como um limite quando $h=0$. Segue diretamente que $\gamma_{f}$ é contínua em todo seu domínio.

Exemplo 2.1. Seja $f:[0,1] \rightarrow \mathbb{R}$ a função identidade. Para cada $y \in \mathbb{S}_{h}(x)$, com $h \in \mathcal{H}$ e $x \in[0,1]$, tem-se $|f(x)-f(y)|^{2}=h^{2}$. Segue através de um cálculo bastante simples que a função $\gamma_{f}$ fica determinada pela lei $\gamma_{f}(h)=h^{2}$. Como esta função é estritamente crescente em $\mathcal{H}$, segue-se que $f$ é $\mu$-espacialmente dependente e o alcance da dependência $\mu$-espacial de $f$ é $\mathfrak{a}=1$ (alcance total).

\section{Dependência $\mu_{n}$-espacial}

O propósito desta seção é, sem que hajam perdas de consistência matemática, discutir uma maneira de viabilizar a aplicação desta teoria à situações práticas.

Pois bem, seja $\mathbb{D}$ uma região do espaço $\mathbb{R}^{m}$ e consideremos suas projeções $\pi_{1}(\mathbb{D}), \ldots, \pi_{m}(\mathbb{D})$ sobre os $m$ eixos coordenados. Como $\mathbb{D}$ é conexo e compacto, a imagem de $\mathbb{D}$ por cada projeção é um intervalo fechado da reta. Sejam $R_{1}, \ldots, R_{m}$ os menores intervalos fechados de extremos inteiros contendo $\pi_{1}(\mathbb{D}), \ldots, \pi_{m}(\mathbb{D})$, respectivamente. Então, $R=R_{1} \times \cdots \times R_{m}$ é o menor retângulo cujos vértices são pontos de coordenadas inteiras, contendo a região $\mathbb{D}$. Dado um inteiro $n>1$, dividamos $R_{1}, \ldots, R_{m}$ em intervalos fechados adjacentes de amplitude $1 / 2^{n}$.

Sejam $J=[b, b+r]$ um intervalo real com extremos inteiro e $n>1 \mathrm{um}$ inteiro arbitrariamente fixado. Denominaremos $n$-ésima partição diática do intervalo $J$ ao conjunto $X_{J}^{n}=\left\{b, b+\frac{1}{2^{n}}, b+\frac{1}{2^{n-1}}, \ldots, b+r\right\}$ que possui $2^{n} r-\sum_{i=0}^{n-1} 2 i$ elementos.

Fixado um inteiro $n>1$, considere as $n$-ésimas partições diádicas dos intervalos 
$R_{1}, \ldots, R_{m}$, ou seja, os conjunto $X_{R_{1}}^{n}, \ldots, X_{R_{m}}^{n}$, respectivamente. Vamos denotar:

$$
X_{\mathbb{D}}^{n}=\left(X_{R_{1}}^{n} \times \cdots \times X_{R_{m}}^{n}\right) \cap \mathbb{D} .
$$

Tal conjunto chamar-se-á $n$-ésimo suporte amostral diádico de $\mathbb{D}$, e denotamos,

$$
\mathcal{H}_{\mathbb{D}}^{n}=\left\{d(x, y): x, y \in X_{\mathbb{D}}^{n}, x \neq y\right\} .
$$

Se $h \in \mathcal{H}_{\mathbb{D}}^{n}$, diremos que $h$ é uma distância factível em $X_{\mathbb{D}}^{n}$.

Consideradas estas notações, definamos a função

$$
\vartheta_{n}: \mathcal{H}_{\mathbb{D}}^{n} \times X_{\mathbb{D}}^{n} \longrightarrow \mathbb{R} \text { pela lei } \vartheta_{n}(h, x)=v_{m-1}\left(\mathbb{S}_{h}(x)\right) \text {. }
$$

Note que a função $\vartheta_{n}$ é a restrição da função volume circular local $\vartheta$ ao subconjunto próprio $\mathcal{H}_{\mathbb{D}}^{n} \times X_{\mathbb{D}}^{n}$ de $\mathcal{H} \times \mathbb{D}$.

Se $x$ é um elemento de $X_{\mathbb{D}}^{n}$, vamos denotar:

$$
\mathcal{H}_{\mathbb{D}}^{n}[x]=\left\{d(x, y): y \in X_{\mathbb{D}}^{n} \backslash\{x\}\right\} \quad \text { e } \quad \dot{\mathcal{H}}_{\mathbb{D}}^{n}[x]=\mathcal{H}_{\mathbb{D}}^{n}[x] \backslash\left\{\max \mathcal{H}_{\mathbb{D}}^{n}[x]\right\} .
$$

É claro que se $n$ e $k$ são dois inteiros positivos e $n \geq k$, então, para todo $x \in X_{\mathbb{D}}^{n}$ tem-se $\mathcal{H}_{\mathbb{D}}^{n}[x] \supset \mathcal{H}_{\mathbb{D}}^{k}[x]$ e $\dot{\mathcal{H}}_{\mathbb{D}}^{n}[x] \supset \dot{\mathcal{H}}_{\mathbb{D}}^{k}[x]$. O seguinte resultado segue diretamente do fato de serem os números diádicos densos na reta.

Lema 3.1. Existe $n$ suficientemente grande tal que $\dot{\mathcal{H}}_{\mathbb{D}}^{n}[x] \neq \emptyset$ para todo $x \in X_{\mathbb{D}}^{n}$.

Lema 3.2. Suponha que $\dot{\mathcal{H}}_{\mathbb{D}}^{n}[x] \neq \emptyset$. Então $\vartheta_{n}(h, x)>0$ para todo $h \in \dot{\mathcal{H}}_{\mathbb{D}}^{n}[x]$.

Demonstração. Seja $h \in \dot{\mathcal{H}}_{\mathbb{D}}^{n}[x]$; então $h<\delta(\mathbb{D})$ e, obrigatoriamente, o conjunto $\mathbb{S}_{h}(x)$ contém um arco não-degenerado. Portanto, $\vartheta_{n}(h, x)>0$.

Doravante denotaremos $\dot{\mathcal{H}}_{\mathbb{D}}^{n}=\mathcal{H}_{\mathbb{D}}^{n} \backslash\left\{\max \mathcal{H}_{\mathbb{D}}^{n}\right\}$ e consideraremos $n$ suficientemente grande de modo que tenhamos $\dot{\mathcal{H}}_{\mathbb{D}}^{n}[x]$ não-vazio para todo $x \in X_{\mathbb{D}}^{n}$. Por conseguinte, teremos também $\dot{\mathcal{H}}_{\mathbb{D}}^{n}$ não-vazio.

Para cada $h \in \mathcal{H}_{\mathbb{D}}^{n}$ denotamos $X_{\mathbb{D}}^{n}[h]=\left\{x \in X_{\mathbb{D}}^{n}: \exists y \in X_{\mathbb{D}}^{n} \operatorname{com} d(x, y)=h\right\}$.

O seguinte resultado é trivial.

Lema 3.3. Sejam $x \in X_{\mathbb{D}}^{n}$ e $h \in \mathcal{H}_{\mathbb{D}}^{n}$. Então $x \in X_{\mathbb{D}}^{n}[h]$ se, e somente se, $h \in \mathcal{H}_{\mathbb{D}}^{n}[x]$.

Lema 3.4. Seja $h \in \dot{\mathcal{H}}_{\mathbb{D}}^{n}$. Então existe $x \in X_{\mathbb{D}}^{n}[h]$ tal que $\vartheta_{n}(h, x)>0$.

Demonstração. Seja $h \in \dot{\mathcal{H}}_{\mathbb{D}}^{n}$. Então, $h \in \dot{\mathcal{H}}_{\mathbb{D}}^{n}[x]$ para algum $x \in X_{\mathbb{D}}^{n}$. Pelo lema anterior, $x \in X_{\mathbb{D}}^{n}[h]$. Além disso $\vartheta_{n}(h, x)>0$, pelo Lema 3.2.

Chamamos volume circular $n$-diádico a função

$$
\mathcal{N}_{n}: \mathcal{H}_{\mathbb{D}}^{n} \rightarrow \mathbb{R} \text { dada por } \mathcal{N}_{n}(h)=\frac{1}{2 \# X_{\mathbb{D}}^{n}[h]} \sum_{x \in X_{\mathbb{D}}^{n}[h]} \vartheta_{n}(h, x) .
$$

Nota 3.1. Nota-se, sem demais esforço, que para cada $h \in \mathcal{H}_{\mathbb{D}}^{n}$, o valor $\mathcal{N}_{n}(h)$ é uma aproximação para $\mathcal{N}(h)$. Além disso, tal aproximação será tanto melhor quanto maior for o inteiro positivo $n$, independentemente da distância factível $h \in \mathcal{H}_{\mathbb{D}}^{n}$. 
Proposição 3.1. Sejam $\mathbb{D} \subset \mathbb{R}^{m}$ uma região e $n$ é um inteiro positivo. Temos:

(a) Se $h \in \dot{\mathcal{H}}_{\mathbb{D}}^{n}$, então $\mathcal{N}_{n}(h)>0$.

(b) Pode ocorrer tanto $\mathcal{N}_{n}\left(\max \mathcal{H}_{\mathbb{D}}^{n}\right)>0$ quanto $\mathcal{N}_{n}\left(\max \mathcal{H}_{\mathbb{D}}^{n}\right)=0$ (dependendo possivelmente da região $\mathbb{D}$ e/ou do inteiro $n)$, sendo que a segunda possibilidade apenas é factivel quando $\max \mathcal{H}_{\mathbb{D}}^{n}=\delta(\mathbb{D})$.

Demonstração. O item (a) segue do Lema 3.4 e da definição da função $\mathcal{N}_{n}$.

Para se provar a sentença (b) basta notar que se $h^{\prime}=\max \mathcal{H}_{\mathbb{D}}^{n}<\delta(\mathbb{D})$, então, para algum ponto $x_{0} \in X_{\mathbb{D}}^{n}\left[h^{\prime}\right]$, tem-se que $\mathbb{S}_{h^{\prime}}\left(x_{0}\right)$ contém um arco não-degenerado.

Agora, o leitor pode considerar uma região $\mathbb{D}$ como sendo um hipercubo no $\mathbb{R}^{m}$ cujos vértices são pontos de coordenadas inteiras, e verificar que este é um exemplo em que $\delta(\mathbb{D})=\max \mathcal{H}_{\mathbb{D}}^{n}$ e $\mathcal{N}_{n}(\delta(\mathbb{D}))=0$ para todo inteiro positivo $n$. Por outro lado, tomando-se $\mathbb{D}$ como o $m$-disco de raio $3 / 4$ centrado na origem do espaço $\mathbb{R}^{m}$, verifícase que $\max \mathcal{H}_{\mathbb{D}}^{1}=\sqrt{2}<\delta(\mathbb{D})$ e $\mathcal{N}_{1}\left(\max \mathcal{H}_{\mathbb{D}}^{1}\right)>0$, enquanto $\max \mathcal{H}_{\mathbb{D}}^{2}=3 / 2=\delta(\mathbb{D})$ e $\mathcal{N}_{2}\left(\max \mathcal{H}_{\mathbb{D}}^{2}\right)=0$.

Observação 3.1. A proposição anterior não implica dizer que se $\max \mathcal{H}_{\mathbb{D}}^{n}=\delta(\mathbb{D})$ então não possamos ter $\mathcal{N}_{n}\left(\max \mathcal{H}_{\mathbb{D}}^{n}\right)>0$. De fato, pode-se construir vários exemplos simples em que $\mathcal{N}_{n}\left(\max \mathcal{H}_{\mathbb{D}}^{n}\right)>0$ embora se tenha max $\mathcal{H}_{\mathbb{D}}^{n}=\delta(\mathbb{D})$. O leitor é convidado a construir um tal exemplo tomando $\mathbb{D}$ como uma região do espaço $\mathbb{R}^{2}$ consistindo de um setor circular de ângulo central inferior a $\pi / 3$ radianos.

Para cada $h \in \mathcal{H}_{\mathbb{D}}^{n}$ vamos, a partir de agora, denotar: $\mathbb{S}_{h}[x]=\mathbb{S}_{h}(x) \cap X_{\mathbb{D}}^{n}$.

Para cada ponto $x \in X_{\mathbb{D}}^{n}$ chamamos $\mu_{n}$-variância circular localizada em $x$ a função

$$
\mathcal{M}_{f}^{n}[x]: \mathcal{H}_{\mathbb{D}}^{n}[x] \rightarrow \mathbb{R} \text { dada por } \mathcal{M}_{f}^{n}[x](h)=\frac{\vartheta_{n}(h, x)}{\# \mathbb{S}_{h}[x]} \sum_{y \in \mathbb{S}_{h}[x]}|f(y)-f(x)|^{2}
$$

Observação 3.2. Pela definição de $\mathcal{H}_{\mathbb{D}}^{n}[x]$ segue-se que $\mathbb{S}_{h}[x] \neq \emptyset$ para todo $h \in$ $\mathcal{H}_{\mathbb{D}}^{n}[x]$, donde $\# \mathbb{S}_{h}[x]>0$ para todo $h \in \mathcal{H}_{\mathbb{D}}^{n}[x]$. Logo, a função $\mathcal{M}_{f}[x]$ está bem definida. Além disso, esta função é não-negativa, e $\mathcal{M}_{f}^{n}[x](h)=0$ se, e somente se, $f(y)=f(x)$ para todo $y \in \mathbb{S}_{h}[x]$, ou $v_{m-1}\left(\mathbb{S}_{h}(x)\right)=0$.

Nota 3.2. Para cada $h \in \mathcal{H}_{\mathbb{D}}^{n}[x]$, o valor $\mathcal{M}_{f}^{n}[x](h)$ pode ser entendido como uma aproximação de $\mathcal{M}_{f}(h, x)$. Além disso, tal aproximação torna-se tanto melhor quanto maior é o inteiro $n$ e menor é a distância factível $h \in \mathcal{H}_{\mathbb{D}}^{n}$.

O Lema 3.3 afirma que se $h \in \mathcal{H}_{\mathbb{D}}^{n}$ então o valor $\mathcal{M}_{f}^{n}[x](h)$ está definido se, e somente se, $x \in X_{\mathbb{D}}^{n}[h]$. Isto nos permite definir o $\mu_{n}$-semivariograma induzido por $f$ como a função

$$
\gamma_{f}^{n}: \dot{\mathcal{H}}_{\mathbb{D}}^{n} \rightarrow \mathbb{R} \text { dada por } \gamma_{f}^{n}(h)=\frac{1}{2 \mathcal{N}_{n}(h)} \sum_{x \in X_{\mathbb{D}}^{n}[h]} \mathcal{M}_{f}^{n}[x](h)
$$

Definição 3.1. Diremos que uma função contínua $f: \mathbb{D} \rightarrow \mathbb{R}$ é $\mu_{n}$-espacialmente dependente se existe uma distância factivel $h \in \dot{\mathcal{H}}_{\mathbb{D}}^{n}, h>1 / 2^{n}=\min \dot{\mathcal{H}}_{\mathbb{D}}^{n}$, tal que 
$\gamma_{f}^{n}$ é estritamente crescente em $\left[1 / 2^{n}, h\right] \cap \dot{\mathcal{H}}_{\mathbb{D}}^{n}$. A maior distância factível $\mathfrak{a}_{n}$ com esta propriedade será denominada alcance da dependência $\mu_{n}$-espacial de $f$.

Exemplo 3.1. Seja $\mathbb{D}=[0,1] \times[0,1]$ região do $\mathbb{R}^{2}$. O conjunto das distâncias factíveis no primeiro suporte amostral diádico $X_{\mathbb{D}}^{1}$ de $\mathbb{D}$ é $\mathcal{H}_{\mathbb{D}}^{1}=\left\{\frac{1}{2}, \frac{\sqrt{2}}{2}, 1, \frac{\sqrt{5}}{2}, \sqrt{2}\right\}$. Seja $f: \mathbb{D} \rightarrow \mathbb{R}$ definida como $f(x, y)=4\left(x^{2}+y^{2}\right)$. Os valores assumidos por $\gamma_{f}^{1}$ em $\mathcal{H}_{\mathbb{D}}^{1}$ estão indicados na tabela abaixo e mostram que a função $f$ é $\mu_{1}$-espacialmente dependente com alcance da dependência $\mu_{1}$-espacial total.

\begin{tabular}{c||c|c|c|c}
$h$ & $\frac{1}{2}$ & $\frac{\sqrt{2}}{2}$ & 1 & $\frac{\sqrt{5}}{2}$ \\
\hline \hline$\gamma_{f}^{1}(h)$ & $\frac{10845}{512}$ & $\frac{91}{2}$ & 116 & 336
\end{tabular}

Para pôr fim a esta seção note-se que as Notas 3.1 e 3.2 resultam no seguinte: Para cada $h \in \dot{\mathcal{H}}_{\mathbb{D}}^{n}$ o valor $\gamma_{f}^{n}(h)$ é uma aproximação para $\gamma_{f}(h)$, e tal aproximação torna-se tanto melhor quanto maior é o inteiro positivo $n$ e menor é a distância factível $h \in \dot{\mathcal{H}}_{\mathbb{D}}^{n}$. Isto implica dizer que para um pequeno intervalo (não-degenerado) $\mathcal{H}_{0}=\left[0, h_{0}\right)$ que intercepta $\dot{\mathcal{H}}_{\mathbb{D}}^{n}$, a curva $\left.\gamma_{f}\right|_{\mathcal{H}_{0}}$ será um bom ajuste para o gráfico discreto $\mathcal{G}_{0}=\left\{\left(h, \gamma_{f}^{n}(h)\right): h \in \dot{\mathcal{H}}_{\mathbb{D}}^{n} \cap \mathcal{H}_{0}\right\}$. Portanto, temos o seguinte:

Teorema 3.1. Seja $f: \mathbb{D} \rightarrow \mathbb{R}$ uma função contínua. Então, $f$ é $\mu$-espacialmente dependente se, e somente se, $f$ é $\mu_{n}$-espacialmente dependente para algum inteiro $n$ suficientemente grande.

\section{Funções Intrinsecamente $\mu$-espaciais}

Definição 4.1. Sejam $\mathbb{D} \subset \mathbb{R}^{m}$ uma região e $f: \mathbb{D} \rightarrow \mathbb{R}$ uma função contínua. Diremos que $f$ é intrinsecamente $\mu$-espacial se, uma vez dada a garantia de que $f$ é $\mu_{n}$-espacialmente dependente para algum inteiro positivo $n$, o mesmo se verificar para uma infinidade de outros inteiros maiores que $n$.

Definição 4.2. Seja $f: \mathbb{D} \rightarrow \mathbb{R}$ uma função intrinsecamente $\mu$-espacial. Diremos que $f$ é $\widetilde{\mu}$-espacialmente dependente se $f$ é $\mu_{n}$-espacialmente dependente para algum inteiro positivo $n$.

O Teorema 3.1 no âmbito destas novas terminologias implica no seguinte:

Teorema 4.1. Seja $f: \mathbb{D} \rightarrow \mathbb{R}$ uma função intrinsecamente $\mu$-espacial. Então, $f$ é $\mu$-espacialmente dependente se, e somente se, $f$ é $\widetilde{\mu}$-espacialmente dependente.

\section{Dependência $\mu_{0}$-espacial: uma Equivalência com a Geoestatística}

Esta última seção do texto destinar-se-á a vislumbrar o leitor da origem das idéias deste trabalho que, na verdade, vieram à generalizar, para o caso de funções definidas em regiões, os conceitos geoestatísticos de dependência espacial até então 
trabalhados no contexto de variáveis regionalizadas definidas e amostradas em subconjuntos finitos no espaço Euclideano de dimensão dois. Deveras, falado isto, nada mais há que se fazer no empreendimento a que se dispõe esta seção a não ser introduzir os conceitos geoestatísticos de dependência espacial. Não obstante, isto será feito no contexto deste trabalho e mantendo-se notações e terminologias aqui inseridas, ficando a cargo do leitor consultar tais conceitos no contexto de variáveis regionalizadas nas notações então apresentadas. (Ver, por exemplo, [2] ou [3]).

Seja $\mathbb{X}$ um subconjunto finito do espaço $\mathbb{R}^{m}$ e suponha que uma função contínua intrinsecamente $\mu$-espacial $f: \mathbb{D} \rightarrow \mathbb{R}$ esteja definida numa região $\mathbb{D}$ do $\mathbb{R}^{m}$ contendo $\mathbb{X}$, de modo que não necessariamente se conheça o valor assumido por $f$ em todos os pontos de $\mathbb{D}$, mas que se conheça o valor $f(x)$ para todo $x \in \mathbb{X}$.

Definimos o conjunto de distâncias factíveis em $\mathbb{X}$ como sendo

$$
\mathcal{H}_{\mathbb{X}}=\{d(x, y): x, y \in \mathbb{X}, x \neq y\} .
$$

Em semelhança a notação já utilizada no texto, vamos denotar, para cada $h \in$ $\mathcal{H}_{\mathbb{X}}$ e cada ponto $x \in \mathbb{X}$, o conjunto $\mathbb{S}_{h}[x]=\partial B_{h}(x) \cap \mathbb{X}$.

Definiremos na seqüência uma série de funções bastante semelhantes as já definidas neste trabalho. Tal semelhança é mantida para que se compreenda a homogeneidade dos conceitos apresentados aqui com os do restante do texto. Na verdade, as definições aqui apresentadas são as mesmas já introduzidas, a menos da medida utilizada; substituímos a medida de Lebesgue pela medida de contagem.

Definamos, inicialmente, a função:

$$
\vartheta_{0}: \mathcal{H}_{\mathbb{X}} \times \mathbb{X} \rightarrow \mathbb{R} \text { por } \vartheta_{0}(h, x)=\# \mathbb{S}_{h}[x]
$$

Agora, para cada $h \in \mathcal{H}_{\mathbb{X}}$, denotemos $\mathbb{X}[h]=\{x \in \mathbb{X}: \exists y \in \mathbb{X} \operatorname{com} d(x, y)=h\}$, e definamos

$$
\mathcal{N}_{0}: \mathcal{H}_{\mathbb{X}} \rightarrow \mathbb{R} \text { por } \mathcal{N}_{0}(h)=\left\lceil\frac{1}{2} \sum_{x \in \mathbb{X}[h]} \vartheta_{0}(h, x)\right\rceil,
$$

onde $\lceil\alpha\rceil$ indica o maior inteiro menor ou igual a $\alpha$. Em termos práticos, para cada $h \in \mathcal{H}_{\mathbb{X}}$, o valor $\mathcal{N}_{0}(h)$ expressa o número de pares de pontos de $\mathbb{X}$ separados pela distância factível $h$.

Para cada ponto $x \in \mathbb{X}$ vamos denotar $\mathcal{H}_{\mathbb{X}}[x]=\{d(x, y): y \in \mathbb{X}\}$, e definir a função

$$
\mathcal{M}_{f}^{0}[x]: \mathcal{H}_{\mathbb{X}}[x] \rightarrow \mathbb{R} \text { por } \mathcal{M}_{f}^{0}[x](h)=\sum_{y \in \mathbb{S}_{h}[x]}|f(x)-f(y)|^{2} .
$$

Definimos, por fim, a função

$$
\gamma_{f}^{0}: \mathcal{H}_{\mathbb{X}} \rightarrow \mathbb{R} \text { por } \gamma_{f}^{0}(h)=\frac{1}{2 \mathcal{N}_{0}(h)} \sum_{x \in \mathbb{X}[h]} \mathcal{M}_{f}^{0}[x](h) .
$$

O leitor pode verificar que no contexto de variáveis regionalizadas intrisecamente estacionárias, esta é a função denominada função semivariograma. Aqui, no entanto, a denominaremos função $\mu_{0}$-semivariograma induzido por $f$. E em semelhança as definições utilizadas naquele contexto e em todo este trabalho, introduzimos a seguinte definição: 
Definição 5.1. Diremos que $f$ é $\mu_{0}$-espacialmente dependente se existe uma distância factivel $h \in \mathcal{H}_{\mathbb{X}}, h>\min \mathcal{H}_{\mathbb{X}}$, tal que $\gamma_{f}^{0}$ é estritamente crescente em $\left[\min \mathcal{H}_{\mathbb{X}}, h\right] \cap \mathcal{H}_{\mathbb{X}}$. A maior distância factível com esta propriedade será denominada alcance da dependência $\mu_{0}$-espacial de $f$ e denotada por $\mathfrak{a}_{0}$.

O leitor há de concordar que esta definição é equivalente àquela que corresponde a dependência espacial para o caso do tratamento de variáveis regionalizadas nos termos da Geoestatística.

Tudo isto mostra que o estudo geoestatístico da dependência espacial de variáveis regionalizadas intrinsecamente estacionárias é um caso especial e particular do estudo da dependência $\mu$-espacial de funções contínuas intrinsecamente $\mu$-espaciais definidas em regiões de espaços Euclideanos, como realizado neste trabalho. A principal particularidade é, de fato, a substituição da medida de Lebesgue pela medida de contagem quando se restringe uma função definida em toda uma região do espaço $m$-dimensional para um subconjunto finito da mesma. O contexto prático da Geoestatística é, ainda, geralmente restrito ao caso 2-dimensional, uma vez que suas aplicações se dão, principalmente, no estudo de fenômenos naturais e, por isso, os domínios das funções estudadas constituem subconjuntos da superfície terrestre, havendo sempre a identificação desta, com um ponto excluído, e o plano Euclideano.

Abstract This work consists of a generalization of the concepts of space dependence brought of the scope of the Geoestatistical to a purely mathematical boarding in the cases where one has defined a continuous function the real values on a domain having consisted of a region of the $m$-dimensional Euclidean space.

\section{Referências}

[1] G.B. Folland, "Real analysis. Modern techniques and their aplications", second edition, Pure and Apllied Mathematics, John Wiley \& Sons, New York, 1999.

[2] P. Goovaerts, "Geostatistics for Natural Resources Evaluation", Applied Geostatistics series. Oxford University Press, Oxford, 1997.

[3] R.A. Olea, "Geostatistical Glossary and Multilingual Dictionary", Studies in Mathematical Geology, Oxford University Press, Oxford, 1991.

[4] H. L. Royden, "Real Analysis", third edition, Macmillan Publishing Company, New York, 1988. 